\title{
ON THE ORIGIN AND ENERGY OF OSCILLATORY EARTHQUAKE
}

$$
\text { WAVES* }
$$

\author{
By C. Y. Fu
}

\begin{abstract}
A CRITICAL survey of the different modes of generation of oscillatory earthquake waves is given. The consequences of the failure of Hooke's law on the dispersion of waves is indicated. The energy of an earthquake is considered from the theory of elasticity with a discussion of Sezawa ${ }^{2} \mathrm{~s}$ result.
\end{abstract}

EARTHQUAKES are usually assumed to be of impulsive origin. Yet the first part of a seismogram often shows a finite train of sustained oscillations instead of a solitary wave. There seems to be a lacuna in the life history of the disturbance which needs to be filled. Apart from repeated faulting, which is itself a multiple source and will naturally lead to a number of waves, the transformation of a pulse into a train of oscillations is commonly attributed to one or more of the following causes: (i) dispersion, (ii) selective absorption, (iii) multiple reflection, and (iv) resonance.

The dispersion of surface waves has been observed, for example, by Gutenberg and Richter, ${ }^{1}$ who made use of this fact for the delineation of the structural units of the earth's crust. But the mechanism of this dispersion as suggested by them is of a particular kind. It must be noted, however, that no appreciable dispersion has been observed in body waves.

There is no doubt that every seismic wave must suffer absorption. Yet the comparison of the amplitudes of $\mathrm{PP}$ and $\mathrm{P}$, or those of $\mathrm{P}$ and $\mathrm{P}^{\prime} \mathrm{P}^{\prime}$, shows that the absorption is extremely small. The observation of the $\mathrm{M}$ and $\mathrm{W}$ phases leads to the same conclusion. That absorption alone is not a sufficient cause has long since been indicated by Jeffreys. ${ }^{2}$ Recently, Ricker ${ }^{3}$ proposed a wavelet theory which was chiefly based on selective absorption. However, his demonstration with respect to artificial earthquakes does not seem to be entirely free from ambiguity, and as for natural earthquakes there is as yet no confirmation.

Both multiple reflection and resonance are not likely to be important. Even granting the most favorable layering of the ground, the waves, as shown by the seismograms of explosions, are oscillatory before any reflection occurs. For waves traveling similar paths, seismograms recorded at the same station do not always show a characteristic frequency. Resonance, even if it exists, must play an insignificant role.

Each one of these causes, be it sufficient in itself or not, provides a mechanism for the transformation of an initial disturbance which is presumed to be a pulse. How this pulse is originally generated is of no consequence. Alternatively, it is also possible that the initial disturbance may simply begin its journey in the form of a

\footnotetext{
* Manuscript received for publication November 4, 1944.

1 B. Gutenberg and C. F. Richter, "On Seismic Waves (Third Paper), " Gerland's Beitr. $z$. Geophysik, 47:73-131 (1936)

2 H. Jeffreys, "On Surface Waves of Earthquakes," Mon. Not. Roy. Astron. Soc., Geophys. Suppl., 1:282-292 (1925)

${ }^{3}$ N. Ricker, "The Form and Nature of Seismic Waves and the Structure of Seismograms, ${ }^{22}$ Geophysics, 5:348-366 (1940); "A Note on the Determination of the Viscosity of Shale from the Measurement of Wavelet Breadth,"2 Geophysics, 6:254--258 (1941); "Further Developments in "the Wavelet Theory of Seismogram Structure,"2 Bull. Seism. Soc. Am., 33:197-228 (1943); "Wavelet Functions and Their Polynomials," Geophysics, 9:314-323 (1944).
} 
finite train of damped waves. Then it is not even necessary that we should interpose a mechanism of transformation.

Generation of initially oscillatory waves.-Consider the following problem: In an infinite elastic medium of density $\rho$ and elastic constants $\lambda$ and $\mu$, is found a spherical cavity of radius $a$. At an initial time $t=0$, a uniform and constant pressure $P_{0}$ is applied to the inner wall of the cavity. This pressure is sustained for a short time $t_{1}$ and is then released. What is the nature of the disturbance generated in the medium outside the cavity? Although the condition of this problem simulates more closely that of an explosion, it is not unreasonable to expect a qualitative similarity from the natural earthquake waves. Since we are only concerned with the initial nature of the disturbance, it is quite sufficient to consider no other discontinuity in the medium than the boundary of the cavity. The problem is a straightforward one, with very simple initial and boundary conditions. Owing to the high degree of symmetry, the wave equation admits of an exact solution. The result as obtained by Kawasumi and Yosiyama ${ }^{4}$ is as follows:

$$
u=-\frac{a^{2} P_{0}}{2 \mu} \sqrt{\frac{\mu}{\lambda+\mu}} \frac{1}{r} e^{-\frac{2 v^{2}}{a V} \tau} \sin \frac{2 v}{a} \sqrt{1-\left(\frac{v}{V}\right)^{2}} \tau+[\ldots]
$$

where $u$ is the radial displacement, $\tau$ the retarded time $=t-(r-a) / V$, and $v$ and $V$ are the velocities of the transversal and longitudinal waves, respectively. The terms in the bracket are of the order of $1 / r^{2}$ and are negligible when $r$ and $t_{1}$ are moderately large. It is seen that this is a damped harmonic wave with

$$
\text { period }=\frac{\pi a}{v \sqrt{1-\left(\frac{v}{V}\right)^{2}}} \text { and damping ratio }=e^{\pi \sqrt{\frac{\mu}{\lambda+u}}}
$$

It is true that this is a highly damped wave and for all practical purposes may be regarded as a pulse. However, it must be noted that (1) is derived under the assumption that the full intensity of $P_{0}$ is applied instantaneously. If the pressure is increased from zero to its full value $P_{0}$ at a finite speed, the damping will be different. In an important extension of the foregoing calculation, Sezawa and Kanai ${ }^{5}$ investigated the disturbance as a function of the speed of application of the pressure. The essential results were that both the maximum amplitude of the displacement and the damping of the wave decrease with the speed.

These calculations, which are based on the theory of small displacements, not only reveal the oseillatory nature of the disturbance, but also admit of other applications. In a series of recent papers, Sharpe ${ }^{6}$ applies these results to explain several

${ }^{4} \mathrm{H}$. Kawasumi and R. Yosiyama, "On an Elastic Wave Animated by the Potential Energy of Initial Strain,"2 Bull. Earthq. Res. Inst., Tokyo Imp. Univ., 13:496-503 (1935).

$5 \mathrm{~K}$. Sezawa, "Elastic Waves Produced by Applying Statical Force to a Body or by Releasing It from a Body," Bull. Earthq. Res. Inst., Tokyo Imp. Univ., 13: 740-748 (1935); K. Sezawa and K. Kanai, "Elastic Waves Formed by Local Stress Changes of Different Rapidities," ibid., 14:10-16 (1936).

6 J. A. Sharpe, "The Production of Elastic Waves by Explosion Pressures, I: Theory and Empirical Field Observations," Geophysics, 7:144-154 (1942); "The Production of Elastic Waves by Explosion Pressures, II : Results of Observations with an Exploding Charge, ${ }^{22}$ Geophysics, 7:311-321 (1942); "The Effect of Charge Size on Reflection Records," Geophysics, $9: 131-142(1944)$. 
interesting phenomena in applied seismology concerning the relations between the nature of the shot and that of the seismic record. The dependence of the period and damping upon the various constants has been indicated above. The variation of amplitude with the speed of the dynamite has been worked out by Sezawa and Kanai and extended by Sharpe. The wave amplitude depends also on the radius of the cavity and the elastic constants of the medium surrounding the shot point, as can be easily seen from expression (1).

It must be pointed out here that the results we have obtained so far refer only to the true ground movements. In making comparison with observations, we must also take account of the nonlinear amplifications of the recording instruments. This effect would be quite prominent in the very high and very low frequency ranges. Consequently, distortions must be expected in the records of sharp pulses and long waves. It is sometimes justifiable to discard these components of the extreme frequency ranges in drawing certain qualitative conclusions. Strictly speaking, when a quantitative comparison is to be made, the principle of superposition, and consequently the Fourier integral theorem, should be used, together with a proper weight factor. But then the resulting integral might be too complicated to evaluate.

When an explosion occurs, the wall of the cavity is inevitably shattered. The idea of an "equivalent cavity," which is vaguely defined as the entire shattered region, is sometimes used. But this leads to a further complication. When the medium is shattered, Hooke's law no longer holds. This failure of Hooke's law would lead to dispersion, as will be shown presently. Thus we shall have not a single train of waves as expressed by equation (1), but a group of such wave trains.

When the displacement is small, the relation between stress and strain is a linear one and the strain energy function of the medium is a homogeneous quadratic fuction of the strain components. When the displacement is large, this is no longer the case. Among other things, the strain energy function may depend upon the higher derivatives of the displacements. Let us, for simplicity, consider only a onedimensional case and assume in particular that the strain energy function $W$ is still a homogeneous quadratic function, but of both the first and the higher derivatives. Let the kinetic energy per unit volume be denoted by $T$. Then

$$
T=1 / 2 \rho\left(\frac{\partial S}{\partial t}\right)^{2}, \quad W=1 / 2 a_{1}\left(\frac{\partial S}{\partial x}\right)^{2}+1 / 2 a_{2}\left(\frac{\partial^{2} S}{\partial x^{2}}\right)^{2}+\ldots
$$

where $S$ is the displacement and the $a$ 's are constants. By Hamilton's principle,

$$
\delta \int_{t_{1}}^{t_{2}} d t \int_{x_{1}}^{x_{2}}(T-W) d x=0
$$

the equation of motion can be written as

$$
\rho \frac{\partial^{2} S}{\partial t^{2}}=\frac{\partial}{\partial x} \frac{\partial W}{\partial S^{\prime}}-\frac{\partial^{2}}{\partial x^{2}} \frac{\partial W}{\partial S^{\prime \prime}}+\frac{\partial^{3}}{\partial x^{3}} \frac{\partial W}{\partial S^{\prime \prime \prime}}-\ldots \quad S^{\prime}=\frac{\partial S}{\partial x}, \quad \text { etc. }
$$

If we substitute a wave form $S=A \cos (\omega t-k x)$ in this last expression, we obtain

$$
\rho \omega^{2}=a_{1} k^{2}+a_{2} k^{4}+a_{3} k^{6}+\ldots
$$


When Hooke's law holds, all the $a$ 's but $a_{1}$ vanish. Then $\omega=\sqrt{a_{1} / p}$ and $\partial \omega / \partial k$ $=\omega / k=$ wave velocity. This means that the medium is not dispersive. But if the other $a$ 's do not all vanish, $\partial \omega / \partial k \neq \omega / k$ and any sinusoidal wave will be dispersed in the vicinity of the source. If $W$ is a more complicated function than here assumed, equation (4) will be more complicated too. In fact, if (4) is a relation between $\omega$ and $k$ other than linear, there will be dispersion.

The amplitude of the wave decreases as the wave advances. The dispersion stops when the displacements are so far reduced that the linear law is restored. It follows from this consideration that a large shock would give rise to a wave train of a larger number of oscillations. Thus we have here another possibility of generating an oscillatory earthquake wave.

So far as is known, the general theory of spherical waves of finite amplitude is not available even for a fluid. The problem is, indeed, very difficult. Not only is the mathematical difficulty almost insurmountable at present, but also the physical nature of the process is not well understood. In this connection, attention may be called to a paper by Unwin ${ }^{7}$ dealing with sound waves of large amplitudes by spherical concentrations of compressed air. A step-by-step method has been developed under the assumption of constant entropy. Several interesting results have been brought out which are not expected from the theory for small amplitudes. One of these is that from a single region of condensation a train of waves is produced instead of a single crest propagated outward. Our qualitative conjecture in the casse of an elastic solid also leads to similar results.

Energy considerations.- In calculating the energy of an earthquake, we often make use of the wave amplitude, or the magnitude scale which is based on the wave amplitude. However, this is only that part of the energy which is released in the form of seismic waves. Although this part is usually of primary interest, it should be distinguished from the total energy transformation which oceurs during an earthquake.

The principal purpose of the work of Kawasumi and Yosiyama ${ }^{8}$ is to prove a hypothesis that the seismic energy released in an earthquake is simply the potential energy which has been stored in the medium. In other words, the elastic wave is animated by the potential energy of initial strain. While this is true under certain conditions, the situation seems to need some further clarification, because the distribution of the different forms of energies when there is an external source of energy supply is different from that when there is no source. In the first case, how much of the total energy goes into the seismic waves depends on the rate at which the energy is supplied, but both the wave and potential energies are derived from the source. In the second case, if other forms of dissipation of energy can be neglected, the whole decrease of potential energy must eventually be released as seismic waves, but the nature of these waves and therefore their observability depend on how the potential energy is released.

In the classical theory of elasticity, we have the following theorem $:^{9}$ The potential energy of deformation of a body which is in equitibrium under given load is equal to

${ }^{7}$ J. J. Unwin, "The Production of Waves by the Sudden Release of a Spherical Distribution of Compressed Air in the Atmosphere,"2 Proc. Roy. Soc. London, A 178: 153-170 (1941).

8 Loc. cit.

A. E. H. Love, Elasticity, 4th ed., Cambridge Univ. Press (1927), pp. 173, 178. See also Sezawa's paper cited. 
half the work done by the external forces acting through the displacements from the unstressed state to the state of equilibrium.

It is implied in this theorem that the load is applied instantaneously and that only the final state is one of equilibrium. Hence, half the work done by the external forces must have been spent as other forms of energy. In an earthquake, these are the wave and heat energies, the latter being usually negligible. On the other hand, if the external forces are applied at so slow a rate that every state of the medium is one of equilibrium, there is no other form of energy obtainable except the strain energy, and the first law of thermodynamics would require that the rate of increase of the energy within a medium must be equal to the rate at which work is done by the traction across its surface. It follows from these considerations that, when external forces are applied to an elastic medium, at most half the work done goes to the seismic waves. This occurs only when the full intensity of the forces is attained at an infinite speed. When the forces are increased from zero to their full intensity at very low speed, no seismic energy is obtained. For a finite speed of application of the forces, the seismic energy would have a value between zero and one-half the work done by the external forces.

We may also describe these results in the following manner: Let forces be applied to a medium and let the intensity of the forces be increased from zero to their final values uniformly. Then the medium will be stressed and at the same time a disturbance or a pulse of certain width will be sent out. The extent of this disturbance or the sharpness of the pulse will depend, among other things, upon the time that is required for the medium to reach its final state. If this time is very large, the waves sent out will be of very long period and therefore cannot be observed.

When there is no source of energy and the strain energy of the medium is in some way released, this energy must be carried away by the seismic waves, provided the heat loss is neglected. Sezawa ${ }^{10}$ contested the view which Kawasumi and Yosiyama set out to prove by pointing out that the amount of seismic energy derived from external forces depends upon the speed at which these are applied. But he also asserted that the same results would apply when the potential energy is released. This part of his argument seems a little obscure. His deduction was based on the following premises: that the release of a statical force $F$ that was being applied to a part of an elastic body is dynamically equivalent to the application of a negative force $-F$, and that the seismic energy is independent of the initial stress. While the second premise is true so long as the principle of superposition holds, the first one does not correspond to the conditions of the previous case, in which the body is supposed to be deformed from its unstressed state. Since the strain energy function is quadratic in the strain components, the change of potential energy does depend on the initial stress.

Except in an explosion or the collapse of a cavity, it is difficult to imagine an instantaneous change of stress of a finite amount in any part of an extended medium. The change must take place at a finite speed. Since in nature the potential energy tends to a minimum, any redistribution of stress would lead to a decrease of potential energy and the balance is carried away by the waves. But not all the energy thus

\footnotetext{
${ }^{10} \mathrm{~K}$. Sezawa, loc. cit., and also "On the Relation between Seismic Origins and Radiated Waves, "Bull. Earthq. Res. Inst., Tokyo Imp. Univ., 14:149-154 (1936).
} 
released can be observed in a seismogram. Earthquakes are simply those waves which are of short enough periods to be felt or recorded. The term "elastic rebound" as used in seismology simply signifies an earth movement which is quick enough to release an appreciable amount of observable wave energy. The relation of this speed to the period of the wave is not easy to find except in the very special and artificial case worked out by Sezawa; but suffice it to say that quicker movement must associate with shorter period. Since in a slow earth movement the potential energy is mainly redistributed and only a very small fraction of this energy goes to the waves, what we can observe really accounts for the main part of the released energy in a sufficiently long period of time.

In applied seismology we are interested in getting the maximum amount of seismic energy from a given amount of dynamite. Then the speed factor may play a very important role in the efficiency of a source. This point has been discussed by Sharpe for the case of the spherical cavity. It is again emphasized here from a general consideration.

I wish to acknowledge my sincere thanks to Professor B. Gutenberg for his constant advice and encouragement. I also wish to thank Mr. H. O. Wood for many valuable suggestions.

Cadifornia Instimute of Technology

Pasadena, California

(Balch Graduate School of the Geological Sciences, Contribution No. 378) 